\title{
Measurements and searches of Higgs boson decays to two quarks with the ATLAS experiment at the LHC
}

\author{
Marco Battaglia ${ }^{a, *}$ and Andrea Sciandra ${ }^{a}$ \\ ${ }^{a}$ Santa Cruz Institute of Particle Physics, University of California at Santa Cruz, \\ High Street, Santa Cruz, CA, USA \\ E-mail: marco.battaglia@ucsc.edu
}

The study of the properties of the Higgs boson is central to the LHC physics program for testing the Standard Model and searching for New Physics signals. Testing the couplings of the Higgs boson to fermions is important for understanding the origin of fermion masses and $H \rightarrow b \bar{b}$ is sensitive to New Physics shifting the relation between the Yukawa coupling and the quark masses. Measurements of Higgs boson decays to quark pairs are dominated by the $b \bar{b}$ state. Having the largest branching fraction, $H \rightarrow b \bar{b}$ controls the Higgs total width and therefore the branching fraction of all other decays. Through efficient flavour tagging, $H \rightarrow b \bar{b}$ is also the main Higgs hadronic decay channel accessible at the LHC and provides us with the best statistics in Higgs processes with small cross section and in corners of the phase space. ATLAS has obtained results for $H \rightarrow b \bar{b}$ production in all the main processes: associated $V H$ production, vector boson fusion (VBF) and gluon-gluon fusion (ggF). These results are presented with particular emphasis on the reconstruction techniques adopted in the resolved and boosted regimes and their interpretation in searches for new phenomena. Results for $t \bar{t} H, H \rightarrow b \bar{b}$ are presented in another contribution to these proceedings.

40th International Conference on High Energy physics - ICHEP2020

July 28 - August 6, 2020

Prague, Czech Republic (virtual meeting)

${ }^{1}$ on behalf of the ATLAS collaboration

* Speaker 
The importance of $H \rightarrow b \bar{b}$ in the study of the Higgs boson profile can hardly be overestimated. Testing the couplings of the Higgs boson to fermions is important for understanding the origin of fermion masses and $H \rightarrow b \bar{b}$ is sensitive to New Physics (NP) effects shifting the relation between the Yukawa coupling and the $b$ quark mass. An example is the $\Delta_{b}$ effect in Supersymmetry[1-3].

The NP effects can be encoded in an effective field theory (EFT). EFTs describe the low-energy remnant of the NP phenomenology (such as new heavy particles or new strong interactions with large energy cut-off) at scales beyond the LHC reach. In particular the ggF process is sensitive to the loop nature of effective $g g H$ coupling, while the $V H$ and VBF processes may help to pin down the tensor structure of the $H V V$ couplings. Constraints on EFT parameters from the analysis of $V H$ production are presented at Section 2.

The dataset collected during the LHC Run 2 makes possible to probe not only the static properties of the Higgs boson, such as the couplings, but also its dynamical properties, such as differential cross sections, in the $b b$ final state. The differential cross section for Higgs boson production as a function of the Higgs transverse momentum, $p_{T}$, provides enhanced sensitivity to $\mathrm{NP}$ models by giving access to new contributions, in particular at high $p_{T}$ [4-8]. Furthermore, the signal-to-background ratio increases with the $p_{T}$ of the candidate hadronic system thus justifying the study of $H \rightarrow b \bar{b}$ processes in bins of $p_{T}$ and the exploration of the high $p_{T}$ region.

The overwhelming QCD background, intrinsic to the hadronic final state, can be reduced by selecting processes where the Higgs boson is produced in association with another particle that can be reliably used for trigger and event selection. This is a $W \rightarrow \ell v$ or a $Z \rightarrow \ell \ell$ (with $\ell=e$, $\mu$ ) boson in the $V H$ associate production, a photon in the VBF+ $\gamma$ and an ISR gluon in the $g g H+$ jet(s) process. The reduction in rate introduced by requiring the production of the additonal particle is more than compensated by the reduction in QCD background and the improvements in event reconstruction. In the case of $g g H+$ jet process, the emission of an hard ISR gluon also provides the Higgs boson with significant transverse momentum.

\section{Reconstruction}

The $H \rightarrow b \bar{b}$ selection in the ATLAS [9] analyses is based on the reconstruction of two $b$-tagged jets. The di-jet opening angle scales as $1 / p_{T}$ inducing a smooth transition from the "resolved" to the "boosted" topology in the Higgs $p_{T}$ range of $450-650 \mathrm{GeV}$. At lower $p_{T}$ the Higgs bosons are reconstructed as a pair of $R=0.4$ calorimetric $b$-tagged jets. At high $p_{T}$ they are a single large-R $(R=1.0)$ jet with variable-radius $(\mathrm{VR})$ track jet substructures reconstructed with a $p_{T}$-dependent radius parameter used for flavour tagging.

Hadronic jets are reconstructed from noise-suppressed topological clusters of calorimeter energy deposits with the anti- $k_{t}$ algorithm [10] using $\mathrm{R}=1.0$ for large- $\mathrm{R}$ jets and 0.4 for small- $\mathrm{R}$ resolved jets. Large- $\mathrm{R}$ jets are groomed using trimming methods to improve jet mass resolution and stability against pile-up conditions. The large- $\mathrm{R}$ jet mass calculation combines tracking and calorimetric information. The mass response is calibrated using $W-, Z$ - and top-enriched samples $[11,12]$. The residual systematics are still a significant source of uncertainties in the extraction of the $H \rightarrow b \bar{b}$ yields. The mass resolution is improved by applying analysis-specific corrections for: i) contributions by muons from heavy hadron semi-leptonic decays ("Muon-in-Jet"), ii) contributions by neutrinos and non-reconstructed particles through the ratio of particle-level 
to reconstructed jet energy ("PtReco") and iii) event kinematic fitting constraining the $b b$ and $\ell \ell$ systems to be balanced in the transverse plane and the di-lepton mass to be consistent with the $Z$ mass by rescaling all objects in the event. These corrections improve the mass resolution at the $H$ peak by factors ranging from $5 \%$ up to $40 \%$, depending on the specific process and kinematics, and shift events from the low mass tail to the bulk of the Higgs boson mass peak.

Flavour tagging combines the response of track- and vertex-based physics taggers into highlevel discriminants with multivariate classifiers. Fixed efficiency working points are defined and the efficiencies for accepting $b, c$ and light jets are measured on calibration data samples used to define appropriate simulation scaling factors and systematic uncertainties[13].

Systematic uncertainties related to the jet mass scale and resolution and to flavour tagging rank among the main sources of experimental systematics in the $V H$, boosted $V H$ and $\mathrm{VBF}+\gamma, H \rightarrow b \bar{b}$ analyses.

\section{Results and Interpretations}

ATLAS has conducted an extensive program of searches and measurements for the $H \rightarrow b \bar{b}$ channel in the main Higgs production processes. The associated $V H(V=W, Z)$ production represents the most sensitive mode for $H \rightarrow q \bar{q}$, due to the $W$ and $Z$ leptonic decays providing energetic leptons for efficient trigger and reduction of the QCD background.

The VBF yields jet-only final states that are difficult to distinguish from non-resonant $b$-quark QCD production. The use of $\mathrm{VBF}+\gamma$ improves both the triggering and the background rejection. The dominant ggF production mode is very challenging due to the large QCD multi-jet background. Events with hard gluon radiation giving significant $p_{T}$ to the Higgs boson have to be selected. Even with this requirement, QCD events with $g \rightarrow b \bar{b}$ gluon splitting still provide the dominant background.

The analyses in the associated $V H$ channel $(V=W, Z)$ categorises events into the 0-, 1- and 2-lepton channels to target $Z H \rightarrow v v b \bar{b}, W H \rightarrow \ell v b \bar{b}$ and $Z H \rightarrow \ell \ell b \bar{b}$. Higgs candidates are reconstructed using two $b$-tagged $R=0.4$ jets in the resolved analysis [14] and a large-R jet with $p_{T}>250 \mathrm{GeV}$ with two $b$-tagged VR jet substructures in the boosted analysis [15]. Both analyses are performed on the full Run 2 data set.

The resolved analysis extracts the signal strength from a simultaneous binned profile likelihood fit to the output of a discriminating BDT for the three lepton channels, two jet categories and three $p_{T}$ regions. The BDT inputs are flavour tagging and kinematical variables for the individual jets and the di-jet system. Two sets of BDTs are trained using the same inputs: one to select the $V H$ signal from the backgrounds and the second to separate the $V Z$ process from both the $V H$ signal and the other backgrounds. The signal regions are supplemented by the corresponding control regions for a total of 42 fit regions. The normalisations of the $V+$ jets and $t \bar{t}$ backgrounds are left free in the fit together with the signal yields.

The result is $\mu_{V H}^{b b}=1.02_{-0.11}^{+0.12}$ (stat.) ${ }_{-0.13}^{+0.14}$ (syst.) for an observed $V H$ signal significance of $6.7 \sigma, 5.3 \sigma$ in the $Z H$ and $4.0 \sigma$ in the $W H$ channels. This result is cross-checked with that obtained by a fit to the di-jet invariant mass weighted by the discriminant MVA output, as in the earlier ATLAS analysis [16], and found to be statistically compatible at the $1.1 \sigma$ level. The main 

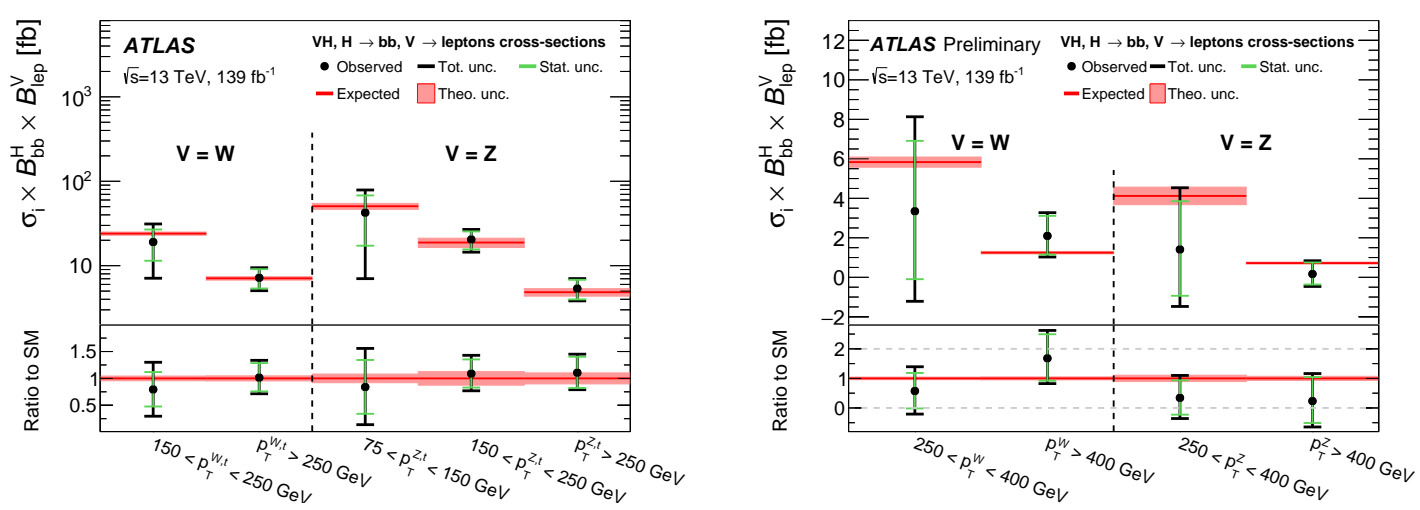

Figure 1: Measured values of the products of production cross section and decay branching fraction in vector boson $p_{T}$ bins from resolved (left) and boosted (right) $V H$ analyses compared to SM expectations (from [14] and [15]).

systematic uncertainties are related to signal modelling, flavour tagging response, jet energy scale calibration and resolution and to the $V+$ jets background modelling.

In the boosted analysis, the result is obtained from a binned maximum-likelihood fit to the large$\mathrm{R}$ jet mass distribution using 10 signal and 4 top control regions. The $V Z(b b)$ and $V H(b b)$ yields are extracted simultaneously obtaining: $\mu_{V Z}^{b b}=0.91 \pm 0.15$ (stat.) ${ }_{-0.17}^{+0.25}$ (syst.) and $\mu_{V H}^{b b}=0.72_{-0.28}^{+0.29}$ (stat.) ${ }_{-0.22}^{+0.26}$ (syst.), respectively, for a $V H$ significance of $2.1 \sigma$. The main sources of systematics are the large-R jet calibration, in particular the mass resolution, and the background modelling. The measured values of the products of production cross section and decay branching fraction in $p_{T}$ bins from the resolved (left) and boosted (right) $V H$ analyses are summarised in Figure 1.

A preliminary result of the search for boosted $H \rightarrow b \bar{b}$ in the $\mathrm{ggF}$ channel was reported for an integrated luminosity of $80.5 \mathrm{fb}^{-1}$ [17]. The analysis is based on the selection of events with two large-R jets: the leading having $p_{T}>480 \mathrm{GeV}$ and two associated $b$-tagged VR jets, and the sub-leading having $p_{T}>250 \mathrm{GeV}$. The dominant QCD contribution is modelled with a 4th-order polynomial exponential function tested on an anti- $b$ tagged validation region, while the rate of the $t \bar{t}$ background contribution is constrained through a dedicated $t \bar{t}$ control region. The $H+$ jet signal and the $Z+$ jet yield are extracted from a binned fit to the large- $\mathrm{R}$ jet mass. The results are $\mu_{V}=1.5$ \pm 0.22 (stat.) ${ }_{-0.25}^{+0.29}$ (syst.) \pm 0.18 (th.) and $\mu_{H}=5.8 \pm 3.1$ (stat.) \pm 1.9 (syst.) \pm 1.7 (th.), where the effect of the QCD contribution is absorbed in the statistical uncertainties and the main sources of systematics are the large- $\mathrm{R}$ jet mass scale and resolution, and the $b$-tagging response.

The study of VBF through the $H \rightarrow b \bar{b}$ channel is based on two complementary search channels: $q q H$ with central and forward jets and the $q q H+\gamma$ production in association with an energetic photon suppressing the gluon-rich, dominant non-resonant $b b j j$ background. The inclusive VBF + jets [18] and the VBF $+\gamma$ [19] analyses both use the full Run-2 statistics.

The VBF $+\gamma$ analysis discriminates the Higgs VBF signal from the backgrounds, dominated by $Z \gamma j j$ and non-resonant $b b \gamma j j$, through an event-level BDT classifier based on a set of kinematic variables chosen to optimise separation while having a low correlation with the mass, to prevent distortions of the di-jet mass spectrum [19]. The BDT output defines three event categories increasingly rich in signal. The Higgs boson rate is extracted from a simultaneous fit to the $b$ - 
tagged di-jet invariant mass. The observed Higgs signal is $\mu_{H}=1.28_{-1.00}^{+1.02}$ for a significance of $1.3 \sigma$, statistically limited. The main experimental systematic contributions are due to the jet energy scale and the flavour tagging uncertainties.

Finally, the sensitivity to $H \rightarrow c \bar{c}$ is still limited due to the much reduced expected branching fraction ( 0.03) compared to the $b b$ channel and the flavour tagging capabilities in an environment that requires reduction of both light-flavoured jets from backgrounds and $b$-jets from Higgs decays. An early ATLAS analysis, carried out the $Z H$ associate production channel on $36 \mathrm{fb}^{-1}$, obtained an observed (expected) upper limit of $\sigma(p p \rightarrow Z H) \times \mathrm{BR}(H \rightarrow c \bar{c})=2.7\left(3.9_{-1.1}^{+2.1}\right) \mathrm{pb}$ at $95 \%$ C.L. corresponding to an observed $\mu$ upper limit of 110 [20].

The corpus of $H \rightarrow b \bar{b}$ results is conducive to several interpretations. First, these results can be combined to determine a constraint to the Higgs coupling to $b$ quarks in terms of the coupling modifier term in the so-called $\kappa$-framework formalism [21]. The result is $\kappa_{b}=0.98_{-0.13}^{+0.14}$ [22].
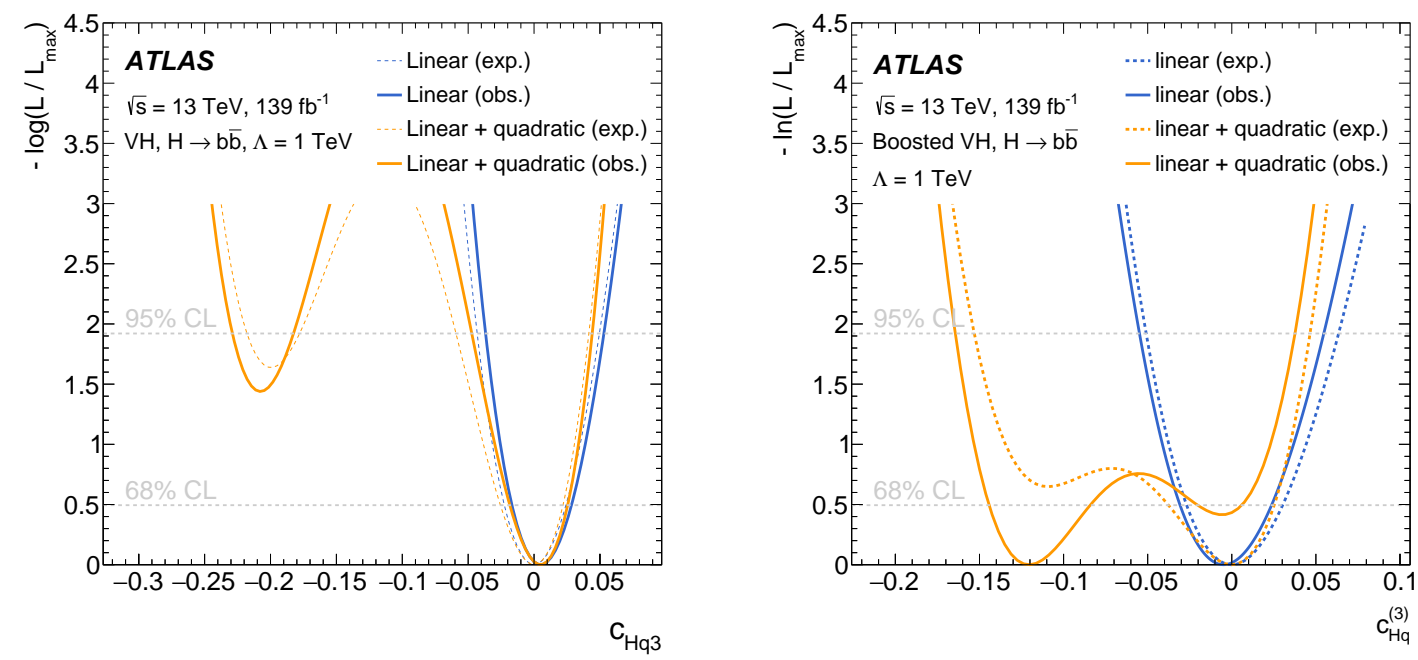

Figure 2: Negative log-likelihood profiles as a function of the variations of the Wilson coefficient of the operator $\mathrm{O}_{\mathrm{Hq}}^{3}$ at the $q q \mathrm{ZH}$ and $q q^{\prime} W H$ production vertices for the resolved (left) and boosted (right) $V H$ analyses (from [14] and [15]). Results obtained with a linear-only parametrisation of the $V H$ production cross section or including linear and quadratic terms into account (orange lines) are compared. Observed and expected results are shown by thick solid and thin dashed lines, respectively.

The measured values of the products of production cross sections and $b \bar{b}$ decay branching fraction in $p_{T}$ bins in the $V H$ channel are interpreted in the context of EFTs in term of constraints to the Wilson coefficients of the relevant operators, or their linear combination. Results using resolved and boosted topologies set constraints on the tensor structure of the process where precision and kinematic reach, respectively, start probing $\Lambda$ scales of order $1 \mathrm{TeV}$. The analysis of the results from the boosted $V H$ topology show a gain in the constraints on the Wilson coefficients by splitting the $p_{T}^{V}$ range in two bins $\left(250<p_{T}^{V}<400 \mathrm{GeV}\right.$ and $\left.p_{T}^{V}>400 \mathrm{GeV}\right)$ due to the $\mathrm{S} / \mathrm{B}$ and sensitivty increase with $p_{T}$. The analysis is based on dimension 6 operators in the effective Lagrangian. The study of the results obtained assuming linear and linear plus quadratic terms describing the dependance of the $V H$ cross section in $p_{T}$ bins on the Wilson coefficients may provide some first 
indications of the role of higher dimension operators, since linear terms from dim. 8 operators are suppressed by the same power of $1 / \Lambda$ as quadratic terms from dim. 8 operatorsas shown in Figure 2.

\section{References}

[1] L. J. Hall, R. Rattazzi and U. Sarid, Phys. Rev. D 50 (1994), 7048.

[2] M. Carena, M. Olechowski, S. Pokorski and C. E. M. Wagner, Nucl. Phys. B 426 (1994), 269.

[3] J. Guasch, W. Hollik and S. Penaranda, Phys. Lett. B 515 (2001), 367.

[4] C. Grojean, E. Salvioni, M. Schlaffer and A. Weiler, JHEP 05 (2014), 022.

[5] A. Biekötter, A. Knochel, M. Krämer, D. Liu and F. Riva, Phys. Rev. D 91 (2015), 055029.

[6] K. Mimasu, V. Sanz and C. Williams, JHEP 08 (2016), 039.

[7] M. Grazzini, A. Ilnicka, M. Spira and M. Wiesemann, JHEP 03 (2017), 115.

[8] A. Banfi, A. Bond, A. Martin and V. Sanz, JHEP 11 (2018), 171.

[9] ATLAS Collaboration, JINST 3 (2008) S08003.

[10] M. Cacciari, G. P. Salam and G. Soyez, JHEP 04 (2008), 063.

[11] ATLAS Collaboration, Eur. Phys. J. C 79 (2019) no.2, 135.

[12] ATLAS Collaboration, ATLAS-CONF-2020-022.

[13] ATLAS Collaboration, Eur. Phys. J. C 79 (2019) no.11, 970.

[14] ATLAS Collaboration, to appear on Eur. Phys. J. C [arXiv:2007.02873 [hep-ex]].

[15] ATLAS Collaboration, submitted to Phys. Lett. B [arXiv:2008.02508 [hep-ex]].

[16] ATLAS Collaboration, Phys. Lett. B 786 (2018), 59.

[17] ATLAS Collaboration, ATLAS-CONF-2018-052.

[18] ATLAS Collaboration, submitted to Eur. Phys. J. C [arXiv:2011.08280 [hep-ex]].

[19] ATLAS Collaboration, submitted to JHEP [arXiv:2010.13651 [hep-ex]].

[20] ATLAS Collaboration, Phys. Rev. Lett. 120 (2018) no.21, 211802.

[21] S. Heinemeyer et al. [LHC Higgs Cross Section Working Group], [arXiv:1307.1347 [hep-ph]].

[22] ATLAS Collaboration, ATLAS-CONF-2020-027. 